\title{
The Kalman-Yakubovich-Popov lemma in a behavioural framework and polynomial spectral factorization
}

\author{
Robert van der Geest \\ University of Twente \\ Faculty of Applied Mathematics \\ P.O.Box 217, 7500 AE Enschede \\ The Netherlands \\ E-mail: R.A.B.vanderGeest@math.utwente.nl
}

\author{
Harry Trentelman \\ University of Groningen \\ Mathematics Institute \\ P.O. Box 800, 9700 AV Groningen \\ The Netherlands \\ E-mail: H.L.Trentelman@math.rug.nl
}

\begin{abstract}
The classical Kalman-Yakubovich-Popov lemma provides a link between dissipativity of a system in state-space form and the solution to a linear matrix inequality. In this paper we derive the KYP lemma for linear systems described by higher-order differential equations. The result is an LMI in terms of the original coefficients in which the dissipativity problem is posed. Subsequently we study the connection between dissipativity and spectral factorization of polynomial matrices. This enables us to derive a new algorithm for polynomial spectral factorization in terms of an LMI in the coefficients of the polynomial matrix.
\end{abstract}

Keywords dissipative systems theory, two-variable polynomial matrices, linear matrix inequalities, polynomial spectral factorization.

\section{Introduction}

The Kalman-Yakubovich-Popov (KYP) lemma is a classical result relating dissipativity of a system in state-space form to the existence of a solution to a linear matrix inequality (LMI). The result was first formulated by Popov [7], who showed that the solution to a certain matrix inequality may be interpreted as a Lyapunov function for the system associated with this inequality, and that the existence of such a Lyapunov function guarantees that the system is dissipative. After that, Kalman [5] and Yakubovich [8] formulated and proved the result in both directions. Later, Anderson [1] derived the result for the multi-input, multioutput case. The KYP lemma has always been recognized as a key result in systems theory, and over the last years it has regained popularity due to the availability of fast numerical routines for solving LMIs.

In this paper we formulate the KYP lemma for linear differential systems, that is, systems described by a set of linear, higher-order differential equations involving the variables associated with the system. Such descriptions are commonly studied in the behavioural approach to linear systems theory, see for instance Willems [11]. This paper is about systems that are dissipative with respect to a supply rate specified in terms of quadratic differential forms (QDFs). Recently, Willems and Trentelman [12] introduced QDFs for specifying dissipativity in a behavioural framework. A QDF is a quadratic function of the variables associated with the system and some of their higher-order derivatives. Therefore QDFs are particularly apt to specify quadratic expressions involving the variables of differential systems.

The line of reasoning in this paper is as follows. First we formulate the KYP lemma for generalized first-order systems, like in Van der Geest and Rantzer [2]. Then we transform the dissipativity problem in a higherorder framework into an equivalent problem for a firstorder system, in terms of the coefficients of the higherorder problem. Plugging this system into the KYP lemma for generalized first order-systems leads to an LMI in terms of the original coefficients in which the higher-order dissipativity problem is posed.

A system is dissipative with respect to a certain supply rate iff there exists a storage function, see Willems [10]. This storage function is related to the solution to the LMI in the KYP lemma. It may be found in [12] that an alternative way of finding storage functions is by performing a polynomial spectral factorization of a polynomial matrix associated with the supply rate. The polynomial spectral factorization problem is challenging in its own right. A collection of algorithms for solving it may be found in Kwakernaak and Šebek [6]. Necessary and sufficient conditions for the existence of a polynomial spectral factorization may. be found in Yakubovich [9]. We use the connection between the KYP lemma and the spectral factorization approach in the last part of this paper to present a new algorithm for polynomial spectral factorization in terms of an LMI in the coefficients of the polynomial matrix.

\section{Dissipativity in a behavioural framework}

This paper is about linear, time-invariant, differential systems, that is, linear, time-invariant, continuous-time 
systems, whose behaviour consists of all solutions $w$ to a linear differential equation of the form

$$
R\left(\frac{d}{d t}\right) w=0
$$

Here $R \in \mathbb{R}^{p \times q}[\xi]$ is a $p \times q$ polynomial matrix in the indeterminate $\xi$ of a certain degree $N$,

$$
R(\xi):=R_{0}+R_{1} \xi+R_{2} \xi^{2}+\cdots+R_{N} \xi^{N} .
$$

A description of the form (1) is called an autoregressive representation, or kernel representation, and its properties are commonly studied in the behavioural approach to linear systems theory. See for instance Willems [11]. The behaviour described by (1) is defined as the set of all $w$ that satisfy the differential equation,

$$
\mathcal{B}:=\left\{w \in \mathcal{C}^{\infty}\left(\mathbb{R}, \mathbb{R}^{q}\right) \text { s.t. (1) }\right\}
$$

Here $\mathcal{C}^{\infty}\left(\mathbb{R}, \mathbb{R}^{q}\right)$ is the set of infinitely often differentiable functions from $\mathbb{R}$ to $\mathbb{R}^{q}$. The system $\mathcal{B}$ represented by (1) is called controllable if for all $w_{1}, w_{2} \in \mathcal{B}$ there exists a $w \in \mathcal{B}$ and a $T \geq 0$ such that

$$
w(t)= \begin{cases}w_{1}(t) & \text { for } t<0, \text { and } \\ w_{2}(t-T) & \text { for } t \geq T\end{cases}
$$

The following result may be found in [11].

Lemma 2.1 The system $\mathcal{B}$ represented by (1) is controllable if and only if the rank of $R(\lambda)$ is the same for all $\lambda \in \mathbb{C}$.

The representation (1) is minimal if the matrix $R$ has full row-rank. It may be found in [11] that every differential system admits a minimal kernel representation. Moreover, two minimal kernel representations with polynomial matrices $R_{1}$ and $R_{2}$ represent the same behaviour iff there exists a unimodular matrix $U$ such that $R_{1}=U R_{2}$.

In Willems and Trentelman [12] it is shown how quadratic expressions in the variables of differential systems are described very adequately using quadratic differential forms. Let $\Phi \in \mathbb{R}_{\mathrm{sym}}^{q \times q}[\zeta, \eta]$ be a symmetric two-variable polynomial matrix in the commuting indeterminates $\zeta$ and $\eta$,

$$
\Phi(\zeta, \eta):=\sum_{0 \leq i, j \leq K} \Phi_{i j} \zeta^{i} \eta^{j},
$$

where the matrices $\Phi_{i j} \in \mathbb{R}^{q \times q}$ satisfy $\Phi_{i j}=\Phi_{j i}^{T}$, and where $K$ is the degree of $\Phi$, i.e., $K$ is the highest power of $\zeta$ and $\eta$ appearing in $\Phi(\zeta, \eta)$. Consider signals $w \in \mathcal{C}^{\infty}\left(\mathbb{R}, \mathbb{R}^{q}\right)$. The quadratic differential form (QDF) associated with $\Phi$ is

$$
Q_{\Phi}(w):=\sum_{0 \leq i, j \leq K}\left(\frac{d^{i} w}{d t^{i}}\right)^{T} \Phi_{i j}\left(\frac{d^{j} w}{d t^{j}}\right) .
$$

The tilde " $\sim$ is used to denote the matrix containing the coefficients of a polynomial matrix or two-variable polynomial matrix. Thus, the coefficient matrix associated with $R$ is

$$
\widetilde{R}:=\left(\begin{array}{llll}
R_{0} & R_{1} & \cdots & R_{N}
\end{array}\right),
$$

and the coefficient matrix associated with $\Phi$ is

$$
\widetilde{\Phi}:=\left(\begin{array}{llll}
\Phi_{00} & \Phi_{01} & \cdots & \Phi_{0 K} \\
\Phi_{10} & \Phi_{11} & \cdots & \Phi_{1 K} \\
\vdots & \vdots & \ddots & \vdots \\
\Phi_{K 0} & \Phi_{K 1} & \cdots & \Phi_{K K}
\end{array}\right) .
$$

Note that

$$
Q_{\Phi}(w)=\left(\begin{array}{c}
w \\
\dot{w} \\
\ddot{w} \\
\vdots \\
w^{(K)}
\end{array}\right)^{T} \widetilde{\Phi}\left(\begin{array}{c}
w \\
\dot{w} \\
\ddot{w} \\
\vdots \\
w^{(K)}
\end{array}\right) .
$$

Let $D\left(\mathbb{R}, \mathbb{R}^{q}\right)$ be the subset of $\mathcal{C}^{\infty}\left(\mathbb{R}, \mathbb{R}^{q}\right)$ consisting of functions that have compact support. The following result from [12] is that dissipativity of a system is equivalent to the existence of a storage function.

Lemma 2.2 Assume that the system $\mathcal{B}$ represented by (1) is controllable. Then the following two statements are equivalent.

a. For all $w \in \mathcal{B} \cap D\left(\mathbb{R}, \mathbb{R}^{q}\right)$,

$$
\int_{-\infty}^{\infty} Q_{\Phi}(w)(t) d t \geq 0
$$

b. There exists a symmetric two-variable polynomial matrix $\Psi$ such that

$$
\frac{d}{d t} Q_{\Psi}(w) \leq Q_{\Phi}(w) \text { for all } w \in \mathcal{B} .
$$

Stated in words, statement $a$ says that the system $\mathcal{B}$ is dissipative with respect to the supply rate $Q_{\Phi}$, and statement $b$ says that the QDF $Q_{\Psi}$ is a storage function for the supply rate $Q_{\Phi}$ on the behaviour $\mathcal{B}$.

\subsection{The KYP lemma for first-order systems}

A special case of a linear differential system is a continuous-time generalized first-order system of the form

$$
G \dot{w}=F w,
$$

where $G$ and $F$ are real-valued, $p \times q$ matrices. Define the behaviour described by (10) as

$$
\mathcal{B}:=\left\{w \in \mathcal{C}^{\infty}\left(\mathbb{R}, \mathbb{R}^{q}\right) \text { s.t. }(10)\right\} .
$$


The set of consistent points of (10) is

$$
W_{0}:=\left\{w_{0} \in \mathbb{R}^{q} \mid \exists w \in \mathcal{B} \text { s.t. } w(0)=w_{0}\right\} .
$$

The representation (10) is called trim if $W_{0}=\mathbb{R}^{q}$.

The following result is the KYP lemma for generalized first-order systems. We have proven this result in Van der Geest and Trentelman [3], using ideas from dissipative systems theory in a behavioural framework [12].

Theorem 2.3 Let $M=M^{T} \in \mathbb{R}^{q \times q}$. Assume that the system $\mathcal{B}$ represented by (10) is controllable, and that the representation (10) is trim. Then the following two statements are equivalent.

a. For all $w \in \mathcal{B} \cap D\left(\mathbb{R}, \mathbb{R}^{q}\right)$,

$$
\int_{-\infty}^{\infty} w^{T}(t) M w(t) d t \geq 0 .
$$

b. There exists a symmetric matrix $P \in \mathbb{R}^{p \times p}$ such that

$$
M+F^{T} P G+G^{T} P F \geq 0 .
$$

Remark Lemma 2.3 is slightly different from the result in Van der Geest and Rantzer [2], where it is not assumed that the representation (10) is trim, but where the matrix $G$ is assumed to have full row-rank. Note that if the system $\mathcal{B}$ is controllable, then the representation (10) is trim if the matrix $G$ has full row-rank.

\section{The KYP lemma for higher-order systems}

We start off this section with a controllable system in kernel representation, and in a few steps we transform it into a generalized first-order system that is controllable and trim. The first-order representation is formulated in terms of the original coefficients of the higherorder system. Consider the system $\mathcal{B}$ with kernel representation

$$
R\left(\frac{d}{d t}\right) w=0
$$

Here $R \in \mathbb{R}^{p \times q}[\xi]$ is a $p \times q$ polynomial matrix of degree $N$, and $w$ is the manifest variable. Let $r_{i}$ denote the $i$ th row of $R$, and let $\rho_{i} \leq N$ denote its row degree, that is, the maximum of the degrees of the entries in row $r_{i}$. Step 1. The first transformation step is to build an 'extended' kernel representation by differentiating some of the constraints. Consider the following polynomial matrix containing the rows and some derivatives of the rows of $R$.

$$
R^{\mathrm{e}}(\xi):=\left(\begin{array}{c}
R_{1}^{\mathrm{e}}(\xi) \\
R_{2}^{\mathrm{e}}(\xi) \\
\vdots \\
R_{p}^{\mathrm{e}}(\xi)
\end{array}\right)
$$

where

$$
R_{i}^{\mathrm{e}}(\xi):=\left(\begin{array}{c}
r_{i}(\xi) \\
\xi r_{i}(\xi) \\
\vdots \\
\xi^{N-\rho_{i}} r_{i}(\xi)
\end{array}\right) .
$$

Note that the 'extended' kernel representation

$$
R^{\mathrm{e}}\left(\frac{d}{d t}\right) w=0
$$

describes the same behaviour as (13).

Step 2. The second transformation step is to put the variable $w$ and all its relevant derivatives into a vector $\ell$

$$
\ell:=\left(\begin{array}{c}
w \\
\dot{w} \\
\ddot{w} \\
\vdots \\
w^{(N)}
\end{array}\right)
$$

This enables us to derive a first-order latent variable representation with external variable $w$ and latent variable $\ell$.

$$
\begin{aligned}
\left(\begin{array}{ll}
I_{N q} & 0
\end{array}\right) \dot{\ell} & =\left(\begin{array}{ll}
0 & I_{N q}
\end{array}\right) \ell, \\
\widetilde{R^{\mathrm{e}}} \ell & =0, \\
w & =\left(\begin{array}{ll}
I_{q} & 0
\end{array}\right) \ell .
\end{aligned}
$$

Here, for given $n, I_{n}$ denotes the $n \times n$ identity matrix, and $R^{\mathrm{e}}$ denotes the coefficient matrix of $R^{\mathrm{e}}$. The dimensions of $\widetilde{R^{e}}$ are $\left(\sum_{i=1}^{p} N+1-\rho_{i}\right)$ by $(N+1) q$. The (manifest) behaviour of this latent variable representation is

$$
\begin{array}{r}
\mathcal{B}_{\text {lv }}:=\left\{w \in \mathcal{C}^{\infty}\left(\mathbb{R}, \mathbb{R}^{q}\right) \mid \exists \ell \in \mathcal{C}^{\infty}\left(\mathbb{R}, \mathbb{R}^{(N+1) q}\right)\right. \text { s.t. } \\
(18),(19),(20)\} .
\end{array}
$$

Note that $\mathcal{B}=\mathcal{B}_{\text {lv }}$, that is, this latent variable representation still describes the same behaviour.

Step 3. The final transformation step is to eliminate the algebraic constraint (19) by rewriting it in image representation,

$$
\widetilde{R^{e}} \ell=0 \Leftrightarrow \ell={\widetilde{R^{e}}}^{\perp} m,
$$

for some vector $m$ of dimension

$$
d=(N+1) q-\sum_{i=1}^{p} N+1-\rho_{i}
$$

where $\widetilde{R}^{\perp}$ is any matrix with $d$ columns such that

$$
\operatorname{ker}\left(\widetilde{R^{\mathrm{e}}}\right)=\operatorname{image}\left(\widetilde{R}^{\mathrm{\perp}}\right) .
$$


This induces the following generalized first-order representation with external variable $w$ and latent variable $m$.

$$
\begin{aligned}
\left(\begin{array}{ll}
I_{N q} & 0
\end{array}\right) \widetilde{R^{\mathrm{e}}} \dot{m} & =\left(\begin{array}{ll}
0 & I_{N q}
\end{array}\right){\widetilde{R^{\mathrm{e}}}}^{\perp} m, \\
w & =\left(\begin{array}{ll}
I_{q} & 0
\end{array}\right) \widetilde{R^{\mathrm{e}}} m .
\end{aligned}
$$

The (manifest) behaviour of this generalized first-order representation is

$$
\mathcal{B}_{\text {gfo }}:=\left\{w \in \mathcal{C}^{\infty}\left(\mathbb{R}, \mathbb{R}^{q}\right) \mid \exists m \in \mathcal{C}^{\infty}\left(\mathbb{R}, \mathbb{R}^{d}\right)\right. \text { s.t. }
$$

$$
\text { (24) and (25) }\} \text {. }
$$

This representation describes the same behaviour as the kernel representation that we started with: $\mathcal{B}=$ $\mathcal{B}_{\text {gfo }}$.

We must ensure that the representation (24) is trim before we can plug it into the KYP lemma for generalized first-order systems. The proof of the following result may be found in [3].

Lemma 3.1 Assume that the matrix $R$ is row-reduced. Then the generalized first-order representation (24) is trim.

A polynomial matrix of full row-rank can be made row-reduced by pre-multiplication with a suitable unimodular matrix, see for instance Kailath [4]. Therefore we may assume without loss of generality that a kernel representation is row-reduced. The main result of this section is formulated as follows. The result may be proven by plugging the generalized first-order system (24) into Theorem 2.3, see Van der Geest and Trentel$\operatorname{man}[3]$.

\section{Theorem 3.2 (KYP lemma)}

Let $\Phi(\zeta, \eta)$ be a symmetric two-variable polynomial matrix as in (5), with $K \leq N$. Assume that the system $\mathcal{B}$ represented by (13) is controllable, and that the matrix $R$ is row-reduced. Then the following two statements are equivalent.

a. For all $w \in \mathcal{B} \cap D\left(\mathbb{R}, \mathbb{R}^{q}\right)$

$$
\int_{-\infty}^{\infty} Q_{\Phi}(w)(t) d t \geq 0
$$

b. There exists a symmetric matrix $P \in \mathbb{R}^{N q \times N q}$ such that

$$
\widetilde{R}^{\perp}{ }^{\perp T}\left(\widetilde{\Phi}+\left(\begin{array}{ll}
0_{N q \times q} & P \\
0_{q \times q} & 0_{q \times N q}
\end{array}\right)+\left(\begin{array}{ll}
0_{q \times N q} & 0_{q \times q} \\
P & 0_{N q \times q}
\end{array}\right)\right) \widetilde{R}^{\mathrm{e}^{\perp}} \geq 0 .
$$

Remark 3.3 There is a one-one relationship between solutions $P$ to the linear matrix inequality in Theorem 3.2 and storage functions $Q_{\Psi}$ for $Q_{\Phi}$ on $\mathcal{B}$, defined by

$$
\widetilde{\Psi}=-P \text {. }
$$

Consequently, these storage functions are of the form

$$
Q_{\Psi}(w)=-\ell^{T}\left(\begin{array}{ll}
P & 0_{N q \times q} \\
0_{q \times N q} & 0_{q \times q}
\end{array}\right) \ell .
$$

Remark It is still possible to use Theorem 3.2 even if the degree $K$ of $\Phi$ is larger than $N$. In this case the degree of the matrix $R$ must first be increased artificially by imposing $R_{N+1}=R_{N+2}=\cdots=R_{K}=$ : $0_{p \times q}$.

\section{Polynomial spectral factorization}

In this last section we present a new algorithm for polynomial spectral factorization. This algorithm is based on the use of a suitable LMI. We begin by introducing some results from factorization theory. A square polynomial matrix $M$ is para-Hermitian if $M^{T}(-\xi)=$ $M(\xi)$. It may be found in for instance Kwakernaak and Šebek [6], that if a para-Hermitian matrix $M$ satisfies

$$
M(i \omega) \geq 0 \text { for all } \omega \in \mathbb{R},
$$

then there exist square polynomial matrices $F(\xi)$ and $C(\xi)$ such that

$$
\begin{aligned}
& M(\xi)=F^{T}(-\xi) F(\xi), \text { and } \\
& M(\xi)=C(\xi) C^{T}(-\xi) .
\end{aligned}
$$

These factorizations are called a symmetric polynomial spectral factorization and spectral co-factorization of $M(\xi)$, respectively. Moreover, if

$$
M(i \omega)>0 \text { for all } \omega \in \mathbb{R},
$$

then $M$ admits a Hurwitz factorization and cofactorization,

$$
\begin{aligned}
& M(\xi)=F_{H}^{T}(-\xi) F_{H}(\xi), \text { and } \\
& M(\xi)=C_{H}(\xi) C_{H}^{T}(-\xi), \text { respectively, }
\end{aligned}
$$

where $F_{H}$ and $C_{H}$ are square and Hurwitz ${ }^{1}$ polynomial matrices.

Consider now a symmetric two-variable polynomial matrix $\Phi$ that satisfies

$$
\Phi(-i \omega, i \omega)>0 \text { for all } \omega \in \mathbb{R} .
$$

Our new spectral factorization technique is to derive an LMI for finding a square and Hurwitz spectral factor $F_{H}(\xi)$ and co-factor $C_{H}(\xi)$ such that

$$
\Phi(-\xi, \xi)=F_{H}^{T}(-\xi) F_{H}(\xi)=C_{H}(\xi) C_{H}^{T}(-\xi) .
$$

Note that this also solves the factorization problem for an arbitrary square, para-hermitian polynomial matrix

\footnotetext{
${ }^{1}$ A square polynomial matrix $H$ is Hurwitz if the real parts of the roots of $\operatorname{det}(H)$ are negative.
} 
$M \in \mathbb{R}^{q \times q}[\xi]$ that satisfies (31), since it is always possible to transform $M$ into $\Phi$ by taking

$$
\Phi(\zeta, \eta):=\frac{1}{2}\left(M^{T}(\zeta)+M(\eta)\right) .
$$

Indeed we have $\Phi(-\xi, \xi)=M(\xi)$, since $M(\xi)$ is paraHermitian.

The following result from [12] gives a characterization of dissipativity in terms of non-negativity of $\Phi(-\xi, \xi)$ along the imaginary axis.

Lemma 4.1 For all $w \in D\left(\mathbb{R}, \mathbb{R}^{q}\right)$,

$$
\int_{-\infty}^{\infty} Q_{\Phi}(w)(t) d t \geq 0
$$

if and only if $\Phi(-i \omega, i \omega) \geq 0$ for all $\omega \in \mathbb{R}$.

This lemma is used in [12] to derive the following result that relates storage functions to spectral factorizations.

Lemma 4.2 (a) Assume that for all $w \in D\left(\mathbb{R}, \mathbb{R}^{q}\right)$,

$$
\int_{-\infty}^{\infty} Q_{\Phi}(w)(t) d t \geq 0
$$

Then there exist storage functions $Q_{\Psi^{-}}$and $Q_{\Psi^{+}}$for $Q_{\Phi}$ such that

$$
Q_{\Psi-} \leq Q_{\Psi} \leq Q_{\Psi+}
$$

for every storage function $Q_{\Psi}$ for $Q_{\Phi}$.

(b) Assume that $\Phi(-i \omega, i \omega)>0$ for all $\omega \in \mathbb{R}$. Let $F_{H}(\xi)$ and $C_{H}(\xi)$ be a square and Hurwitz spectral factor and co-factor of $\Phi(-\xi, \xi)$, respectively. Then

$$
\begin{aligned}
& \Psi^{-}(\zeta, \eta)=\frac{\Phi(\zeta, \eta)-F_{H}^{T}(\zeta) F_{H}(\eta)}{\zeta+\eta}, \text { and } \\
& \Psi^{+}(\zeta, \eta)=\frac{\Phi(\zeta, \eta)-C_{H}(\eta) C_{H}^{T}(\zeta)}{\zeta+\eta}
\end{aligned}
$$

The following theorem is our result on polynomial spectral factorization. The result may be proven by using Lemma 4.2 together with the connection between storage functions and solutions to the linear matrix inequality that is described in Remark 3.3, see [3].

\section{Theorem 4.3 (Spectral factorization)}

Let $\Phi(\zeta, \eta)$ be a symmetric two-variable polynomial matrix of degree $K$, as in (5). Consider the following $L M I$ in the symmetric $K \dot{q} \times K q$ matrix $P$,

$\widetilde{\Phi}+\left(\begin{array}{ll}0_{K q \times q} & P \\ 0_{q \times q} & 0_{q \times K q}\end{array}\right)+\left(\begin{array}{ll}0_{q \times K q} & 0_{q \times q} \\ P & 0_{K q \times q}\end{array}\right) \geq 0$.

Let $L(P)$ denote the left-hand side of this LMI, and define its solution set as

$$
\mathcal{S}:=\left\{P=P^{T} \in \mathbb{R}^{K q \times K q} \text { s.t. } L(P) \geq 0\right\} .
$$

Assume that $\Phi(-i \omega, i \omega)>0$ for all $\omega \in \mathbb{R}$. Then $\mathcal{S}$ is non-empty. Moreover, there exist matrices $P^{-}, P^{+} \in \mathcal{S}$ such that $P^{-} \leq P \leq P^{+}$for all $P \in \mathcal{S}$. Furthermore,

$$
\operatorname{rank}\left(L\left(P^{-}\right)\right)=\operatorname{rank}\left(L\left(P^{+}\right)\right)=q .
$$

As a consequence, there exist $\widetilde{V} \in \mathbb{R}^{q \times(K+1) q}$ and $\widetilde{Q} \in$ $\mathbb{R}^{(K+1) q \times q}$ such that

$$
L\left(P^{+}\right)=\widetilde{V}^{T} \widetilde{V} \quad \text { and } \quad L\left(P^{-}\right)=\widetilde{Q} \widetilde{Q}^{T} .
$$

Define now

$$
\begin{aligned}
& V(\xi):=\widetilde{V}\left(\begin{array}{c}
I \\
\xi I \\
\vdots \\
\xi^{K} I
\end{array}\right), \text { and } \\
& Q(\xi):=\left(\begin{array}{llll}
I & -\xi I & \cdots & \left.(-\xi)^{K} I\right) \widetilde{Q} .
\end{array}\right.
\end{aligned}
$$

Then $V(\xi)$ and $Q(\xi)$ are a square and Hurwitz factor and co-factor of $\Phi(-\xi, \xi)$, respectively.

Example We try our LMI-based algorithm for polynomial spectral factorization in practice on the square, polynomial matrix

$$
M(\xi)=\left(\begin{array}{cc}
-\xi^{2}+1 & -\xi+1 \\
\xi+1 & 2
\end{array}\right)
$$

This matrix is para-Hermitian, since $M^{T}(-\xi)=M(\xi)$. Furthermore,

$$
M(i \omega)=\left(\begin{array}{cc}
\omega^{2}+1 & -i \omega+1 \\
i \omega+1 & 2
\end{array}\right)>0 \text { for all } \omega \in \mathbb{R}
$$

It follows that $M$ admits a Hurwitz factorization and co-factorization. In fact it is easy to find out by inspection for this example that

$$
\begin{aligned}
M(\xi) & =\left(\begin{array}{cc}
-\xi+1 & 0 \\
1 & 1
\end{array}\right) \cdot\left(\begin{array}{cc}
\xi+1 & 1 \\
0 & 1
\end{array}\right)=: F_{H}^{T}(-\xi) F_{H}(\xi) \\
& =\left(\begin{array}{cc}
1 & \xi \\
1 & -1
\end{array}\right) \cdot\left(\begin{array}{cc}
1 & 1 \\
-\xi & -1
\end{array}\right)=: C_{H}(\xi) C_{H}^{T}(-\xi)
\end{aligned}
$$

We derive this factorization using the steps of our LMIbased approach to polynomial spectral factorization.

Step 1. Construct the symmetric two-variable polynomial matrix

$$
\begin{aligned}
\Phi(\zeta, \eta) & :=\frac{1}{2}\left(M^{T}(\zeta)+M(\eta)\right) \\
& =\left(\begin{array}{cc}
-\frac{1}{2} \zeta^{2}-\frac{1}{2} \eta^{2}+1 & -\frac{1}{2} \zeta-\frac{1}{2} \eta+1 \\
\frac{1}{2} \zeta+\frac{1}{2} \eta+1 & 2
\end{array}\right) .
\end{aligned}
$$

Note that $\Phi(-i \omega, i \omega)=M(i \omega)>0$ for all $\omega \in \mathbb{R}$. The degree of $\Phi$ is $K=2$, and the dimension of $\Phi$ is $q=2$. The coefficient matrix of $\Phi$ is

$$
\widetilde{\Phi}=\left(\begin{array}{cccccc}
1 & 1 & 0 & -\frac{1}{2} & -\frac{1}{2} & 0 \\
1 & 2 & \frac{1}{2} & 0 & 0 & 0 \\
0 & \frac{1}{2} & 0 & 0 & 0 & 0 \\
-\frac{1}{2} & 0 & 0 & 0 & 0 & 0 \\
-\frac{1}{2} & 0 & 0 & 0 & 0 & 0 \\
0 & 0 & 0 & 0 & 0 & 0
\end{array}\right) .
$$


Step 2. Let $P$ be a symmetric, $K q$ by $K q$ matrix,

$$
P:=\left(\begin{array}{cccc}
p_{1} & p_{2} & p_{4} & p_{7} \\
p_{2} & p_{3} & p_{5} & p_{8} \\
p_{4} & p_{5} & p_{6} & p_{9} \\
p_{7} & p_{8} & p_{9} & p_{10}
\end{array}\right)
$$

Consider the linear matrix inequality

$$
\left(\begin{array}{cccccc}
1 & 1 & p_{1} & -\frac{1}{2}+p_{2} & -\frac{1}{2}+p_{4} & p_{7} \\
1 & 2 & \frac{1}{2}+p_{2} & p_{3} & p_{5} & p_{8} \\
p_{1} & \frac{1}{2}+p_{2} & 2 p_{4} & p_{5}+p_{7} & p_{6} & p_{9} \\
-\frac{1}{2}+p_{2} & p_{3} & p_{7}+p_{5} & 2 p_{8} & p_{9} & p_{10} \\
-\frac{1}{2}+p_{4} & p_{5} & p_{6} & p_{9} & 0 & 0 \\
p_{7} & p_{8} & p_{9} & p_{10} & 0 & 0
\end{array}\right) \geq 0 .
$$

Because of the zero block in the lower right corner of the LMI, $p_{5}=p_{6}=p_{7}=p_{8}=p_{9}=p_{10}=0$ and $p_{4}=1 / 2$. This implies that $p_{3}=0$ and $p_{2}=1 / 2$. It follows that the solution set to the LMI is

$$
\mathcal{S}:=\left\{\left(\begin{array}{cccc}
p_{1} & \frac{1}{2} & \frac{1}{2} & 0 \\
\frac{1}{2} & 0 & 0 & 0 \\
\frac{1}{2} & 0 & 0 & 0 \\
0 & 0 & 0 & 0
\end{array}\right) \text { s.t. } 0 \leq p_{1} \leq 1\right\} .
$$

Step 3. Factorize

$$
\begin{aligned}
L\left(P^{+}\right) & =\left(\begin{array}{llllll}
1 & 1 & 1 & 0 & 0 & 0 \\
1 & 2 & 1 & 0 & 0 & 0 \\
1 & 1 & 1 & 0 & 0 & 0 \\
0 & 0 & 0 & 0 & 0 & 0 \\
0 & 0 & 0 & 0 & 0 & 0 \\
0 & 0 & 0 & 0 & 0 & 0
\end{array}\right) \\
& =\left(\begin{array}{ll}
1 & 0 \\
1 & 1 \\
1 & 0 \\
0 & 0 \\
0 & 0 \\
0 & 0
\end{array}\right)\left(\begin{array}{llllll}
1 & 1 & 1 & 0 & 0 & 0 \\
0 & 1 & 0 & 0 & 0 & 0
\end{array}\right)=: \tilde{V}^{T} \tilde{V},
\end{aligned}
$$

and

$$
\begin{aligned}
L\left(P^{-}\right) & =\left(\begin{array}{llllll}
1 & 1 & 0 & 0 & 0 & 0 \\
1 & 2 & 1 & 0 & 0 & 0 \\
0 & 1 & 1 & 0 & 0 & 0 \\
0 & 0 & 0 & 0 & 0 & 0 \\
0 & 0 & 0 & 0 & 0 & 0 \\
0 & 0 & 0 & 0 & 0 & 0
\end{array}\right) \\
& =\left(\begin{array}{cc}
1 & 0 \\
1 & -1 \\
0 & -1 \\
0 & 0 \\
0 & 0 \\
0 & 0
\end{array}\right)\left(\begin{array}{cccccc}
1 & 1 & 0 & 0 & 0 & 0 \\
0 & -1 & -1 & 0 & 0 & 0
\end{array}\right)=: \widetilde{Q} \widetilde{Q}^{T} .
\end{aligned}
$$

Define

$$
V(\xi):=\widetilde{V}\left(\begin{array}{c}
I \\
\xi I \\
\vdots \\
\xi^{K} I
\end{array}\right)=\left(\begin{array}{cc}
\xi+1 & 1 \\
0 & 1
\end{array}\right)
$$

and

$$
Q(\xi):=\left(\begin{array}{llll}
I & -\xi I & \cdots & (-\xi)^{K} I
\end{array}\right) \widetilde{Q}=\left(\begin{array}{cc}
1 & \xi \\
1 & -1
\end{array}\right)
$$

It turns out that $V$ and $Q$ are indeed a Hurwitz spectral factor and co-factor of $M$, respectively.

\section{References}

[1] B.D.O. Anderson, 'A system theory criterion for positive real matrices,' SIAM J. Control, vol. 5, 1967, pp. 171-182.

[2] R.A.B. van der Geest and A.R. Rantzer, 'Quadratic performance of generalized first-order systems,' Proc. 35th IEEE CDC, Kobe, Japan, 1996, pp. 4533-4534.

[3] R.A.B. van der Geest and H.L. Trentelman, 'The Kalman-Yakubovich-Popov lemma in a behavioural framework,' accepted for publication in Systems and Control Letters, 1997.

[4] T. Kailath, Linear Systems, Prentice-Hall, 1980.

[5] R.E. Kalman, 'Lyapunov functions for the problem of Lur'e in automatic control,' Proc. Nat. Acad. Sci. USA, vol. 49, 1963, pp. 201-205.

[6] H. Kwakernaak and M. Šebek, 'Polynomial $J$ spectral factorization,' IEEE Trans. Automatic Control, vol. 39, 1994, pp. 315-328.

[7] V.M. Popov, 'Absolute stability of nonlinear systems of automatic control,' Automation and Remote Control, vol. 22, 1961, pp. 857-875.

[8] V.A. Yakubovich, 'Solution of certain matrix inequalities in the stability theory of nonlinear control systems,' Soviet Math. Dokl., vol. 3, 1962, pp. 620623 (translated from Doklady Akademii Nauk SSSR, vol. 143, 1962, pp. 1304-1307).

[9] V.A. Yakubovich, 'Factorization of symmetric matrix polynomials,' Soviet Math. Dokl., vol. 11, 1970, pp. 1261-1264 (translated from Doklady Akademii Nauk SSSR, vol. 194, 1970, pp. 532-535).

[10] J.C. Willems, 'Dissipative dynamical systems Part I: general theory, Part II: linear systems with quadratic supply rates,' Archive for Rational Mechanics and Analysis, vol. 45, No. 5, 1972, pp. 321-393.

[11] J.C. Willems, 'Paradigms and puzzles in the theory of dynamical systems,' IEEE Trans. Automatic Control, vol. 36, 1991, pp. 259-294.

[12] J.C. Willems and H.L. Trentelman, 'On quadratic differential forms,' accepted for publication in SIAM J. Control and Optimization, 1997. 